\title{
Review on Relay Node Selection for Wireless Network
}

\author{
Prashant Kumar Shukla \\ PhD Scholar \\ Dr. K.N.Modi University \\ Newai, Rajasthan
}

\author{
Akhilesh Tiwari, PhD \\ Associate Professor \\ MITS,Gwalior, M.P.
}

\begin{abstract}
Wireless network is one of the most prior network veils in today's times. It is being widely because of its easy development and low cost of access. IEEE 802.11 is one of the known standard which provides various transmission rates such as IEEE $802.11 \mathrm{a}, \mathrm{b}, \mathrm{g}$ and because of their multiple support for transmission rates enhance the usage of WLAN. In this paper, we describes about the protocols which is being used for relay selection. Relay node is the node which supports transmission from source to destination and overcome the problems of traditional methods in terms of throughput, delay, power and so on.
\end{abstract}

\section{Keywords}

WLAN, Cooperative Network, Relay Node

\section{INTRODUCTION}

We ask that authors follow some simple guidelines. In essence, we ask you to make your paper look exactly like this document. The easiest way to do this is simply to download the template, and replace the content with your own material.

The IEEE standard 802.11 i.e. WLAN is widely used in wireless access because of its easy development and low cost of maintenance. This standard describes multiple MAC protocol which is being provided to the nodes for sharing of the channel. The basic method avails for increment the capacity of wireless LAN in-order to provide higher rate for transmission over the physical layer. Multiple access rates are been standardize to expand the physical layer which results in providing higher rates of transmission. There are many features supporting relay node selection mechanism. The IEEE standard 802.11 provides transmission from source to destination but likely with the unfavorable conditions results in poor throughput and low performance for WLAN network. Thus, to overcome these situations we used intermediate node known as relay node which help in complete transmission of packets from source to destination.

But, the condition arising for the selection of relay node with which method, mechanism and criteria the relay node must be selected. Thus, in this paper we provide the description of protocols for relay node selection on following factors:

- Throughput and Delay

- MAC overheads and Retransmission

- Power aware and Efficiency

- Multiple Channel

- Time and Channel Constraint

\section{THROUGHPUT AND DELAY BASED PROTOCOL}

This section contains the description of the protocols based on throughput and delay constraints. It includes following protocols:

\subsection{CoopMAC protocol (2005)}

Wireless LAN supports various rate of transmission, this makes station to experiences degraded quality of channel and to reduce bit error rate for each transmission it uses least rate of transmission. This method leads to throughput fairness anomaly among station with good quality and on contrary. This anomaly degrades the performance of the network in terms of throughput.

The MAC protocol is being proposed to improvises the anomaly been arisen. This protocol is being based upon efficient cooperative schemes. This principle supervises the station with low rate with the assistance of high rate station as helper and with this method low rate station also becomes capable to transmit in a high speed.

CoopMAC protocol supports potential helper acts as an intermediate station between the source and destination at the higher rate rather than the direct transmission. In this protocol, the source do not directly send the packet but transmit the data in two hop using intermediate station as helper. The basic advantage of this protocol makes faster transmission and thus overall time for transmission is being reduced. The helper station takes the data from source and then re-transmission takes place to the destination.

Thus every station maintains Coop Table for all helper. CoopMAC protocol follows five-way handshake and maintain Coop table. This protocol thus improvises the source station transmission suffering from poor quality of the channel and selects the helper station as relay and makes faster transmission to destination.

\subsection{CRS (2006)}

As the wireless protocol mediates between physical layer and the higher layer protocols and the IEEE standard 802.11 supports the feature on both sides. CRS is acronym for Cooperative Relay Service. CRS is being used for WLAN supporting multiple channel rates. It provide that the station with higher rate relays the frames as alternative between the neighboring nodes having low channel rates and access point to enhances the energy efficiency and throughput. CRS cope with the delay as uses the following energy for data forwarding. It results in improvising the throughput without degrading the energy efficiency. CRS proves out to be significant as it enhances the performances for both stations have low and high channel rate. CRS with multiple rates selects the mobile station in cooperative form from local network to avoid degradation in channel rate of transmission. It utilizes the energy efficiency which increases the performances. The usage of channel time in CRS and 
supporting mechanism provides help in forwarding of data packets.

To analyze this protocol we include following:

1. Time allocated for channel

2. Analysis of performance gain

3. Analysis of allocation of channel for forwarding.

CRS system model has three components which help in maintaining the channel energy and throughput:

- Algorithm for selection of proxy station

- Algorithm for scheduling of channel

- Multiple-forward hopping algorithm.

\subsection{SOLOR (2014)}

In the current scenario of IEEE standard 802.11 suffers from the problem known as rate anomaly which results in three major points for consideration such as:

1. Relay addition incremented consumption of power.

2. Variety of throughput and their preferences.

3. Dealing with legacy nodes.

To overcome all these three major problems in the current situation, we have SOLOR viz. Self- Optimizing, LegacyCompatible Opportunistic Relaying which enhance the network performance. It basically works on following criteria such as topology i.e. nodes associated in relaying and relay schedule i.e. time stamp between their access points. It considerably improvises the network throughput performances and reduces the power consumption even it contain legacy nodes i.e. devices present in current network. It attains its goal via selection of topology i.e. the path between access point and station also with the time duration being spent over relay activities.

But these two conditions overcome simply and provide best of throughput and reduced power consumption. It is also capable of compacting with the legacy nodes. This is also being achieved through the method of selecting the path reaching to the access point via relay stations which is known as topology of the network.

Thus, SOLOR consider the adaptable topology to ensure of receiving MCS rates helping in enhancing performance of the network with both parameters of throughput and power consumption.

\section{MAC OVERHEADS AND RETRANSMISSION BASED PROTOCOL}

This section contains the description of protocols which are based on MAC overheads and re-transmission:

\section{1 rPCF (2004)}

Discussing the IEEE 802.11 standard in terms of multiple transmission rates, then to maintain its performance we use rPCF i.e. relay enabled PCF. The PCF based MAC defined used to overcome the problems being arisen due to multi- rate capability. Discussing over channel platform of each node, rPCF utilizes the multi- hops for data transmission through MAC layer which improvises the performance of the system in terms of throughput and delay in transmission considering the negligible control overheads.
As IEEE 802.11 works over the various data rates with respect to various channel conditions, thus packets will be transmitted faster with the help of relay than with direct link which degrades the quality as well as rate. The results of simulation being done over rPCF it outperforms in terms of throughput and transmission.

\subsection{PCF (2005)}

In heterogeneous system, the WLAN plays a vital role and its performance reflects over the complete system. The IEEE standard 802.11 provides two specific MAC mechanisms such as:

- Distributed Coordinated function

- Point Coordinated function.

The PCF is based on polling i.e. selection of nodes function through majority supports. PCF is being designed over the DCF mechanism and hence provides contention free MAC, and thus because of this feature in PCF, it resulted in providing higher throughput and committed services. This is very essential for the real time application. PCF can be implemented our non-real time services. The performance of PCF is being enhances through various efficient and robust polling mechanism. PCF also robust in efficient power management and hence increase the efficiency of whole working system.

\subsection{DCF (2005)}

WLAN provides multiple rates capability in a physical layer and hence this capability degrades performance of the network. Thus, we require the MAC layer supports method to overcome this anomaly. MAC layer mechanism provides the distributed point function in order to maintain the performance of the whole system. DCF is one of the protocol based on carrier sense multiple access with collision avoidance i.e. CSMA/CA. This protocol was being proposed and created for improvising the performance of contention oriented channel used for access. The DCF uses broadly two methods for transmission of data viz.:

1. Basic Access, also known as Default access mechanism.

2. Optional Request to send/ Clear to send.

This protocol works on the principle of handshaking. The basic access mechanism follows two-way handshake method. In this the data is being sent from source and acknowledgement is being send to source from destination.

On the other hand, Optional method uses four- way handshake. In this source send the request for sending of the data and if the receiver is free to receive then send clear to send message and then data is being sent from source station to destination and then to confirm the transmission acknowledgement is being sent on the sender side. This method checks the channel and then reserves the channel till the whole transmission is completed.

Thus it reduces the retransmission of same data and also degrades the MAC overheads leading better performance of the network.

\subsection{CoopMAC (2007)}

Wireless LAN supports various rate of transmission, this makes station to experiences degraded quality of channel and to reduce bit error rate for each transmission it uses least rate of transmission. This method leads to throughput fairness anomaly among station with good quality and on contrary. 
This anomaly degrades the performance of the network in terms of throughput.

The MAC protocol is being proposed to improvises the anomaly been arisen. This protocol is being based upon efficient cooperative schemes. This principle supervises the station with low rate with the assistance of high rate station as helper and with this method low rate station also becomes capable to transmit in a high speed.

CoopMAC protocol supports potential helper acts as an intermediate station between the source and destination at the higher rate rather than the direct transmission. In this protocol, the source do not directly send the packet but transmit the data in two hop using intermediate station as helper. The basic advantage of this protocol makes faster transmission and thus overall time for transmission is being reduced. The helper station takes the data from source and then re-transmission takes place to the destination.

Thus every station maintains Coop Table for all helper. CoopMAC protocol follows five-way handshake and maintain Coop table. This protocol thus improvises the source station transmission suffering from poor quality of the channel and selects the helper station as relay and makes faster transmission to destination.

\section{POWER-AWARE PROTOCOLS}

This section contains the protocol description based on the power efficiency and utilizes the energy in order to enhance the network performance. It includes following protocols:

\subsection{ECTP (2007)}

Energy Efficiency is the one of the major objective in the WLAN performance. Here, we introduce the protocol called ECTP viz. Energy Efficient Cut-Through Protocol. This protocol enhances the energy efficiency of the network in the WLAN. It is a combinational concept of MAC and next hop address without any information about host protocol and provides a relay node in the pair of communication ends adopts a higher data rate on the basis of path loss and channel's condition.

This protocol is the enhancement of MAC protocol which has new means to message exchange techniques communication with relay node. The basic idea for this protocol is that it follows four-way handshake for communication and then relay won't be competitive to channel again. This would save the bandwidth because again competing for channel waste bandwidth for channel which is already being reserved.

\section{MULTI-CHANNEL BASED PROTOCOL}

This section contains the description of the protocol based on multiple channel utilization:

\subsection{Net COOP (2009)}

Earlier design of the network for relay cooperation system results the better throughput but it degrades the lifetime of the network. To improve the life of the network we use Net Coop protocol whose objective is to increment the life time of network with high throughput. This can be achieved by the cooperation of multiple relays. Net Coop algorithm checks and validates the relays which provide best possible cooperation so as to obtain network with high lifetime.

In this protocol, every station has table which records the data rate for the exchange of control message among various pairs of stations and the station which find that the transmission is not for them, then switch themselves to sleep mode. An algorithm for this protocol is applying dual filter to choose the relay node as it selects the node on the basis of throughput improvising and also energy efficient constraints.

This protocol makes the source to choose the transmission mode will be least affected by the relaying and here it is found that the node which is low in energy then the source chooses alternative which results in lowering the loss of energy.

The principle of Net Coop forms by the cooperative format which forms between network throughput and lifetime. It uses flexible strategy for cooperation which supports network performance and enhances lifetime of the network without affecting the throughput.

\section{TIME AND CHANNEL BASED PROTOCOLS}

This section contains the description of the protocol which is based on the time and channel constraints. It includes the following protocols:

\subsection{TOR (2007)}

Functioning over real time environment i.e. the streaming of audio- visual over the wireless is one of the significant technologies emerging in the multimedia types of transmission. The IEEE 802.11 stadard protocol is best suited for traffic handling but for streaming for audio- visual faces following challenges:

- Multi-path Fading

- Communication in gray zone

- Interference

- Time Constraint

- $\quad$ Predictive- Coded Property

To resolve the above described challenges, we present TOR i.e. Time - aware Opportunistic Relay which operates on the MAC layer. This protocol over rules the path diversity to improvise quality of end - to - end service. Basically, relay node simultaneously forward packet to enhance reliability on end - to - end basis. To describe the transmission in time based manner, relay deadline is being calculated for each packet being transmitted.

This deadline is being used by relay node to check the status of the packet. This protocol results in the increment in packet delivery rate. It basically adopts time based transmission for video traffic at each node.

TOR uses the multiple path joints on nodes for over hear the traffic from other nodes, thus it provides higher gain and improves performance of the network. Relay candidate nodes over hear the transmission and relay makes the packet failed if its acknowledgement is missing.

\subsection{TRCCL (2014)}

IEEE Standard for WLAN supports various data rates which provides WLAN capacity increment but also results in anomaly problem. Thus, to emphasis over the problem we propose TRCCL protocol. TRCCL is an acronym for Transmission Rate and Channel Contention - Level protocol. This protocol chooses a relay node by keeping transmission rate as priority.

It also selects the relay node by the transmission being occurred in the neighbor. Here, we use the contention level of 
the channel for judging the transmission of its neighboring nodes.

TRCCL protocol uses the two - level relay selection property which include selection of node and then the exchanging of packets. It estimates the time of transmission by using its rate and select the node which has the least rate of transmission time as relay node. It also checks the contention level of channel which reduces the channel anomaly problem.

Every node in this type maintains a table called as Relay Table. Relay table keep the records for the transmission being shared from source to destination. The protocol, TRCCL selects the optimal relay as a node by taking into account the both condition as contention level of channel and transmission time. For this the results can be obtained through relay table.

The transmission time of the packet can be defined as the time taken by a packet from source node to destination which includes total time with all relays used as an intermediates. While contention level is describe as the probability of the collision. Thus, it outperforms from the previous protocol for the time and channel category in terms of throughput and delay.

\subsection{Comparison of protocols}

\begin{tabular}{|c|c|c|c|}
\hline PROTOCOLS & YEAR & PRINCIPLE/ WORKING & FUTURE RESEARCH \\
\hline PCF & 2003 & $\begin{array}{l}\text { It selects the relay node on the basis of majority supports. } \\
\text { It makes the selection of nodes and organized polling and } \\
\text { result of it node is being selected. }\end{array}$ & $\begin{array}{l}\text { Dynamic polling being organized } \\
\text { to select node so as utilizes the } \\
\text { same energy. }\end{array}$ \\
\hline DCF & 2003 & $\begin{array}{l}\text { It performance is based on the CSMA/CA mechanism. It } \\
\text { selects the node in terms of contention oriented channel. }\end{array}$ & $\begin{array}{l}\text { Selection of the channel based on } \\
\text { low contention and low collision } \\
\text { probability. }\end{array}$ \\
\hline $\mathrm{rPCF}$ & 2004 & $\begin{array}{l}\text { It selects the node for transmission for multiple rates } \\
\text { which is being based on channel condition and uses multi } \\
\text { - hops for data transmission. }\end{array}$ & $\begin{array}{l}\text { rPCF can be used over the } \\
\text { reduction of Control overheads of } \\
\text { MAC. }\end{array}$ \\
\hline COOPMAC & $\begin{array}{c}2005 \text { and } \\
2007\end{array}$ & $\begin{array}{l}\text { It supports low rate station being assisted by high rate } \\
\text { station using intermediate station as helper node to } \\
\text { transmit data from source to destination. }\end{array}$ & $\begin{array}{l}\text { Dynamic performance over static } \\
\text { condition of the Coop MAC } \\
\text { protocol. }\end{array}$ \\
\hline CRS & 2006 & $\begin{array}{l}\text { It selects the relay node from the neighboring nodes } \\
\text { which has higher rate to enhance the energy efficiency of } \\
\text { the low rate node. }\end{array}$ & $\begin{array}{l}\text { Selection of relay node can be } \\
\text { done dynamically from the nodes } \\
\text { come in between transmission. }\end{array}$ \\
\hline ECTP & 2007 & $\begin{array}{l}\text { It uses the combinational concept of MAC and next hop } \\
\text { in order to selects the relay node which saves bandwidth } \\
\text { as it won't compete for reserve channel. }\end{array}$ & $\begin{array}{l}\text { Checking path loss condition and } \\
\text { selects relay to save energy. }\end{array}$ \\
\hline TOR & 2007 & $\begin{array}{l}\text { It uses time constraint for the selection of relay node. For } \\
\text { this it provide deadline to every node to check the status } \\
\text { of the transmitted packet. }\end{array}$ & $\begin{array}{l}\text { Efficient relay node selection to } \\
\text { avoid duplicate receiving of } \\
\text { packets. }\end{array}$ \\
\hline Net COOP & 2009 & $\begin{array}{l}\text { It checks and validates the relay to provide best } \\
\text { cooperation in the network. It uses dual filter to select } \\
\text { relay node based on throughput and energy constraint }\end{array}$ & $\begin{array}{l}\text { Auto updating of the relay table is } \\
\text { required so the selection of the } \\
\text { relay can be done in minimum } \\
\text { time. }\end{array}$ \\
\hline SOLOR & 2014 & $\begin{array}{l}\text { It selects the relay node on basis of topology being used } \\
\text { and the path being selected for the transmission of data } \\
\text { packets. }\end{array}$ & $\begin{array}{l}\text { Selection prior relay through } \\
\text { election algorithm and routing. }\end{array}$ \\
\hline TRCCL & 2014 & $\begin{array}{l}\text { It used two - level for the relay node selection i.e. time } \\
\text { constraint and contention level of the channel. Every node } \\
\text { in this type maintains a table called relay table which } \\
\text { keep records for transmission from source to destination. }\end{array}$ & $\begin{array}{l}\text { Checking of the node before } \\
\text { selecting as relay to avoid } \\
\text { multiple selection of the same } \\
\text { node for transmission. }\end{array}$ \\
\hline
\end{tabular}

\section{CONCLUSION}

IEEE 802.11 standard supports various protocols which outperforms their tasks in order to improvise the performance of the network. These protocols make WLAN, a best suited medium for the transmission of data from source to destination with the help of cooperative communication which includes relay node. These protocols help the source station to choose the relay node so as to enhance the network performance in terms of throughput, delay, MAC overheads and re- transmission, time and channel and efficient - energy constraints.

\section{REFERENCES}

[1] Stefano, Marco, Giordano and Stojmenovic, "Mobile adhoc networking- The cutting-edge directions", Johnwiley, 2013.

[2] Wornell and Laneman, "An efficient protocol for realizing cooperative diversity in wireless networks", IEEE ISIT, 2001, page 294. 
[3] Cao and Zhu, "rDCF- A relay enabled MAC protocol for wireless ad-hoc networks", IEEE Trans. Mobile Computing, vol.5, no.9, pp. 1201-1214, September 2006.

[4] Khalid, Wang and Sankar, "Two relay based cooperative MAC protocol for wireless ad- hoc networks", IEEE Trans, 2011, pp:3361-3373.

[5] Liu, Zhang and Leung, "Residual energy aware cooperative transmission (REACT) in wireless Networks", 19th WOCC, China, 2010, pp 1-6

[6] Hyung, Choi, Chung and Lee, "Performance evaluation of binary negative:- Exponential backoff algorithm in IEEE 802.11 WLAN,” Springer, pp: 294-303, 2006.

[7] Cao, Bin, Yu Ge, Chee Wee Kim, Gang Feng, Hwee Pink Tan and Yun Li, "An experimental study for interuser interference mitigation in wireless body sensor networks", IEEE sensors journal, 13.10.2013: 35853595 .

[8] Qing F. Zhou, Yonghui Li, F.C.M. Lau and Branka Vucetic, "Decode-and-forward Two-way Relaying with Network Coding and Opportunistic Relay Selection," IEEE Transactions on Communications, vol. 58, no. 11, pp. 3070-3076, November 2010.

[9] Biao Han, Jie Li and Jinshu Su, "Optimal relay node placement for multi-pair cooperative communication in wireless networks", Wireless Communications and Networking Conference (WCNC), 2013 IEEE, pp 4724 4729.

[10] Elzbieta Beres and Raviraj Adve, "Optimal Relay-Subset Selection and Time-Allocation in Decode-and-Forward Cooperative Networks", Wireless Communications, IEEE Transactions on Vol:9, Issue:7, 2010, pp 2145 2155.

[11] Yonghui Li, Zihuai Lin and Vucetic, B.,"Joint networkchannel code design for real wireless relay networks", Turbo Codes and Iterative Information Processing (ISTC), 2010 6th International Symposium, 2010, pp 429 $-433$.

[12] Yifan Li, Ping Wang, Niyato, D. and Weihua Zhuang, "A dynamic relay selection scheme for mobile users in wireless relay networks", INFOCOM, 2011 Proceedings IEEE, 2011, pp 256 - 260.

[13] Helmut Adam and Christian Bettstetter, "Adaptive relay selection in cooperative wireless networks", IEEE TRANSACTIONS ON MOBILE COMPUTING, 2013.

[14] Yaming Luo, Jun Zhang, and Khaled B, "Relay Selection for Energy Harvesting Cooperative Communication Systems", Global Communications Conference (GLOBECOM), 2013 IEEE, pp 2514 - 2519

[15] Shan Chu and Xin Wang, "MIMO-Aware Routing in Wireless Mesh Networks", INFOCOM, 2010 Proceedings IEEE, pp 1-9

[16] Zhong Zhou,Shengli Zhou, Jun-Hong Cui and Shuguang Cui, "Energy-Efficient Cooperative Communication Based on Power Control and Selective Single-Relay in Wireless Sensor Networks", Wireless Communications, IEEE Transactions, Vol:7, Issue:8, 2008 pp 3066 - 3078.

[17] Xiaowen, Chandra shekhar and Zhang, "Opportunistic Co-operative Networking-: To Relay or Not To Relay",
Communications, IEEE, Vol:30, Issue:2, 2012 pp 307 314.

[18] Bletsas, A., Hyundong Shin and Win, M.Z., "Cooperative Communications with Outage-Optimal Opportunistic Relaying", Wireless Communications, IEEE Transactions, Vol:6, Issue:9, 2007, pp 3450 - 3460.

[19] Pei Liu, Zhifeng Tao and Panwar, "A cooperative MAC protocol for wireless local area networks", Communications, ICC-2005. IEEE International Conference, Vol:5, 2005, pp 2962 - 2968.

[20] Guo, L., Xiaoning Ding, Haining Wang and Qun Li, "Cooperative Relay Service in a Wireless LAN", Selected Areas in Communications, IEEE Journal, Vol:25, Issue:2, 2007, pp 355 - 368.

[21] Garcia-Saavedra, Rengarajan, Serrano, Camps-Mur, and Costa-Perez, "SOLOR: Self-Optimizing WLANs With Legacy-Compatible Opportunistic Relays", Networking, IEEE/ACM Transactions, 2015, Vol:23, Issue:4 ,pp: $1202-1215$

[22] H. Zhu and G. Cao, "On improving the performance of IEEE 802.11 with relay-enabled PCF" Mobile Netw. Appl (MONET), vol. 9, no. 4, 2005, pp. 423-434.

[23] Hao Zhu and Guohong Cao, "rDCF: A Relay-enabled Medium Access Control Protocol for Wireless Ad Hoc Networks" INFOCOM, IEEE, 2005, Vol:1, pp:12-22.

[24] Hao Zhu, Guohong Cao, "On improving the performance of IEEE 802.11 with multihop concepts", Computer Communications and Networks, 2003, ICCCN 2003, pp:151-156.

[25] Pei Liu, Zhifeng Tao and Narayanan, S. Korakis,"CoopMAC: A Cooperative MAC for Wireless LANs", Selected Areas in Communications, IEEE Journal, Vol:25, Issue:2, 2007, pp 340-354.

[26] Jain-Shing Liu and Lin, C., "ECTP: An EnergyEfficiency Label-Switching MAC Protocol for Infrastructure Wireless Networks", Vehicular Technology, IEEE Transactions, 2007, Vol:56, Issue:3, pp:1399-1417.

[27] Mei-Hsuan Lu, Steenkiste, P. and Tsuhan Chen, "TimeAware Opportunistic Relay for Video Streaming Over WLANs", Multimedia and Expo, 2007 IEEE International Conference, 2007, pp: 1782-1785.

[28] Sunmyeng Kim, "Relay Node Selection Algorithm for Cooperative Communications in Wireless Networks", World Academy of Science, Engineering and Technology, International Journal of Computer, Electrical, Automation, Control and Information Engineering, Vol:8, No:3, 2014, pp:450-453.

[29] Md Helaluddin ahmed, Choong Seon Hong and Sungwon Lee, "Energy efficient smart relay based cooperative MAC for wireless networks", Computing, Springer, February 2014, Volume 96, Issue 2, pp 133 161.

[30] Issari yakul and Ekram Hossain, "Introduction to Network Simulator-2", Springer-Technology \& Engineering, 2011

[31] http://www.isi.edu/nsnam/ns/ 
[32] J. Laneman, G. Wornell and D. Tse, "An efficient protocol for realizing cooperative diversity in wireless networks," in Proc. IEEE ISIT, Jun. 2001, p.294

[33] A. Sendonaris, E. Erkip, and B. Aazhang, "User cooperation-diversity:- System description," IEEE
Transaction Communication, vol. 51, no. 11, 2003, pp. 1927-1938.

[34] Wireless LAN: Medium Access Control (MAC) and Physical Layer (PHY) Specifications- High-Speed Physical Layer Extension in the $2.4 \mathrm{GHz}$ Band, IEEE Std. 802.11b-1999, 1999. 